\title{
Efecto citotóxico del colorante alimentario rojo 40 en nauplios de Artemia salina Leach
}

\author{
Cytotoxic effect of red 40 food dye in Artemia salina nauplii \\ José Mostacero-León ${ }^{1}$, (1D Rafael Mendoza Rodríguez¹ , (D) Priscilla Seijas-Bernabé1 \\ Luis Taramona-Ruíz ${ }^{2}$, (DD Anthony De La Cruz-Castillo , (D) Carlos Gonzales Sarmiento
}

'Universidad Nacional de Trujillo. Trujillo, Perú

${ }^{2}$ Universidad Le Cordon Bleu. Lima, Perú

Recibido: 10/04/2020 Revisado: 20/05/2020 Aceptado: 21/09/2020 Publicado: 16/12/2020

\section{RESUMEN}

Diversos colorantes artificiales empleados en la industria alimentaria, lejos de satisfacer los requerimientos organolépticos de la población; merman en gran medida la salud de quienes lo consumen; tal es el caso del rojo 40 ampliamente utilizado en el Perú. Dentro de este contexto se realizó este estudio preliminar cuyo objetivo principal fue determinar el efecto citotóxico del colorante alimentario rojo 40 en nauplios de Artemia salina. Para ello, se instaló un sistema de incubación de nauplios, para luego ser expuestos a diferentes concentraciones del colorante en estudio, conformando los tratamientos: T0: 0, 00 (mg/L), T1: 3,125 mg/L, T2: 15,625 mg/L y T3: $31,25 \mathrm{mg} / \mathrm{L}$ y finalmente medir su mortandad cada 24 horas por un periodo de 2 días. Se reportó que T0 presentó $0 \%$ de letalidad, el T1 presentó una letalidad del $60 \%$, el T2 una letalidad del $100 \%$ y el T3 una letalidad del $100 \%$. Se concluye que el colorante alimentario rojo 40 presentó un efecto citotóxico e hiperactividad en la locomoción en nauplios de Artemia salina, con una LC 50 de 2.6. Palabras clave: Citotoxicidad, colorante alimentario, rojo 40, Artemia salina.

\begin{abstract}
Recent research supports the fact that many of the dye-type food additives used in the food industry, far from satisfying the organoleptic requirements of the population; greatly reduce the health of those who consume it; such is the case of red 40 widely used in Peru. Based on what has been described, this research focused on determining the cytotoxic effect of red food dye 40 in Artemia salina nauplii. For this, a nauplos incubation system was installed, to then be exposed to different concentrations of the dye under study, forming the treatments: T0: 0.00 (mg / L), T1: $3.125 \mathrm{mg}$ / L, T2: $15.625 \mathrm{mg} / \mathrm{L}$ and T3: $31.25 \mathrm{mg} / \mathrm{L}$ and finally measure their mortality after 48 hours. The results showed that T0 had a $0 \%$ lethality, T1 had a $60 \%$ lethality, T2 a $100 \%$ lethality and T3 a 100\% lethality. It is concluded that the red food dye 40 presented a cytotoxic effect and hyperactivity in locomotion in nauplii of Artemia salina, with an LC 50 of 2.6.
\end{abstract} Keywords: cytotoxicity, food coloring, red 40, Artemia salina. 


\section{INTRODUCCIÓN}

Los aditivos de tipo colorantes son fundamentales en la elaboración de alimentos, ya sea por razones tecnológicas, o para satisfacer los requerimientos organolépticos de la población; ya que forman parte del alimento como un todo y mantiene una estrecha relación con el sabor, aroma y la textura (Santillán et al., 2018; Secco, 1994). Según Okafor et.al (2016); considera que el empleo de este tipo de aditivos es para mantener la apariencia original del alimento después de la etapa de procesamiento (temperaturas altas) y almacenamiento ya que suele modificarse por los factores ntrínsecos (enzimas) y extrínsecos como humedad, luz, radiación, tiempo de anaquel entre otros por lo que cambia la uniformidad y tono del color incluso se considera que puede mantener el sabor y ser una pantalla protectora de vitaminas que son susceptibles a la luz y por último incrementan la aceptabilidad del alimento (Okafor et al.,2016; Shanmugasundaram et al., 2019).

La FDA (Food and Drug Administration) define colorante o pigmento, a cualquier sustancia química obtenida por síntesis o artificio similar, extraída o derivada, con o sin intermediarios del cambio final de identidad, a partir de un vegetal, animal, mineral u otra fuente cuya función principal es impartir color a la comida, medicamentos o cosméticos, es decir, es capaz de impartir color por sí misma (Badui-Dergal, 2006).

Químicamente para ser considerado un pigmento o aditivo colorante debe poseer ciertas características tales como absorber la luz en el espectro visible (400- $700 \mathrm{~nm})$, poseer al menos un grupo cromóforo, tener un sistema conjugado es decir una estructura que alterne con simples y dobles enlaces y por último presentar una resonancia de electrones permitiendo una estabilidad en los componentes orgánicos (Okafor et al.,2016)

En tanto que la clasificación se da entre dos grandes grupos los colorantes naturales y los artificiales, estos últimos son muy empleados porque presentan mayor estabilidad en los productos además de abaratar costos, entre los más utilizados en la industria alimentaria se encuentran amarillo 5, amarillo 6, taratrazina y rojo 40 o Allura. Este último ha causado controversia en cuanto a sus efectos tóxicos, y su uso fue prohibido en algunos países como Francia, Bélgica, Suiza, Inglaterra y Dinamarca, ya que demostraron en diversos ensayos in vitro e in vivo que inducen aberraciones cromosómicas y otros efectos genotóxicos y mutagénicos en mamíferos, debido a que es un colorante azoico (Sal disódica del ácido 6-hidroxi-5-[(2-metoxi-5-metil-4sulfofenil)azo]-2-naftalensulfónico.), además puede provocar intolerancia en aquellas personas alérgicas con ácido salicílico; otro efecto negativo, es que produce la liberación de histamina, y puede intensificar los síntomas del asma; así mismo está implicado en la producción de hiperactividad en niños (Martine et al., 2009; Shanmugasundaram et al., 2019).

Sin embargo, estos colorantes artificiales son los más utilizados en la industria de alimentos, tales como bebidas gasificadas amarillas y anaranjadas, bocaditos de tipo chizitos, chocolates, entre otros (ASPEC,2009; Tripathy y Rao, 2015) que presentan un consumo excesivo e indiferente, por lo que la DIGESA normativizó su uso, solicitando consignar su presencia o ausencia en la etiqueta de los productos, al final de la lista de ingredientes, en forma específica, clara, visible, e indubitable, con letra mayúscula y negrita. La presencia de los colorantes sintéticos en los productos es netamente estética para hacerlos un poco más "brillantes y atractivos" y de 
esta forma favorecer su elección y consumo, especialmente por los niños (DIGESA, 2013).

Si bien ya se ha demostrado ampliamente que el alto consumo de alimentos densamente energéticos, con cantidades elevadas ya sea de azúcar, grasa o sal, provocan obesidad, deterioro en los hábitos de alimentación, adicción y malnutrición en general; ahora enfrentamos otro riesgo: el impacto de estos productos en el comportamiento, especialmente en la población infantil (McCann et al., 2007; CSPI, 2011; DIGESA,2013). Dentro de este contexto, es importante el estudio del colorante rojo 40, ya que, según los antecedentes reportados, el colorante ha causado controversia en cuanto a sus efectos tóxicos, tales como efectos genotóxicos, mutagénicos e inducciones cromosómicas (Abdellah et al.,2015; Amaringo y del Socorro, 2018).

Para realizar estudios y determinar los efectos de estos colorantes en la salud, se emplea bioensayos de manera preliminar, empleando diversos sistemas in vivo, por lo que siempre hay búsqueda de los modelos de test empleando diversos organismos, para que sea útil deben ser ensayos de bajo costo, que los resultados presenten confiabilidad estadística, que sean factibles y rápidos en los resultados además de reproducibles. Siendo los más empleados los microcrústaceos tales como Daphnia sp. Y Artemia spp. Los cuales se han empleado para la determinación de los efectos tóxicos o letales de principios bioactivos de extractos vegetales en la industria farmacéutica, en el aspecto ecológico se han utilizado para estudios de ecotoxicidad en diversos ecosistemas producidos por contaminantes químicos tales como metales pesados, plaguicidas entre otros. En relación a la industria alimentaria estos tipos de test se han utilizado poco principalmente en pruebas con respecto a aditivos (Pino y Jorge ,2010).

Por lo expuesto, se propuso este estudio preliminar cuyo objetivo principal fue determinar el efecto citotóxico en nauplios de Artemia salina, además de proponer un bioensayo o test para predecir la toxicidad a nivel preclínico (laboratorio) de bajo costo y fácil acceso.

\section{MATERIALES Y MÉTODOS}

\section{Instalación del sistema de incubación y preparación del material biológico}

Los huevos o quistes de Artemia salina Leach. se procedieron a incubar en un litro de solución preparada con sal marina al 3,8 \% en un sistema acondicionado con los factores para la respectiva eclosión como temperatura a $250 \mathrm{C}$, concentración de oxígeno de $2.5 \mathrm{mg} / \mathrm{L}, \mathrm{pH} 8$ además con fotoperiodo de 48 horas luz y una zona oscura empleada para la realización de la recolección de los individuos que ya eclosionaron ( nauplios) pues éstos presentan fototropismo positivo, de tal manera que los nauplios se dirigieron al punto de luz y los quistes que no eclosionaron se quedaron en la zona oscura.

\section{Ensayo de citotoxicidad del colorante rojo 40}

Se conformaron 4 grupos experimentales según las concentraciones evaluadas del colorante : T0: $0,0 \mathrm{mg} / \mathrm{L}$ (testigo), T1: 3,125 $\mathrm{mg} / \mathrm{L} ; \mathrm{T} 2: 15,625 \mathrm{mg} / \mathrm{L}$ y T3: 31,25 mg/L; cada sistema se conformó por tubo vial con 10 organismos en solución salina incubados a temperatura ambiente con la adición de la concentración del colorante según el grupo experimental así como se colocó una gota de suspensión de levadura (3 mg de levadura seca en $5 \mathrm{ml}$ de agua salada), para la respectiva alimentación de los nauplios. Se realizaron 3 réplicas por cada tratamiento. 
El primer día de ensayo se realizó un monitoreo cada 4, 6, 8, 12 y 24 horas, con la finalidad de observar si los individuos se aclimataron al medio. Se reportó la tasa de mortalidad a las 24 horas y a las 48 horas (periodo en que terminó el ensayo). Se considera un nauplio muerto cuando no se observa movimiento de sus apéndices por un tiempo de 10 segundos observados al microscopio óptico a $40 \mathrm{X}$.

\section{Análisis estadístico}

El modelo estadístico empleado fue el diseño aleatorizado y la prueba de análisis de varianza (Anova) empleando el programa InfoStat.

\section{RESULTADOS Y DISCUSIÓN}

En cuanto al ensayo de citotoxicidad, la letalidad en el T0 (grupo control o testigo) fue de un $0 \%$ (tabla 1); no se observó alteraciones en el comportamiento de los nauplios. Por otro lado, en los 3 tratamientos problema; se logró apreciar que algunas larvas, presentaban dificultades en la natación a partir de la $12 \mathrm{~h}$, dato que fue aumentando al finalizar el ensayo; de allí que a las $24 \mathrm{~h}$, el porcentaje de letalidad fue del 60\% (en el T1) , 100\% (en el T2) y $100 \%$ (en el T3\%); presentándose a su vez, una DLC de 2,6 mg/ml (figura 1); y en donde se comprobó la existencia de diferencias significativas (Tabla 2); siendo los porcentajes más letales las comprendidas entre los tratamientos T2 y T3 (Tabla 1); comportamiento que ratifica el empleo de nauplios de Artemia salina, en ensayos de citotoxicidad, debido a su gran sensibilidad adquirida, por presentar una delgada cutícula que permite fácilmente el ingreso de diversos compuestos químicos a su interior, alterando su locomoción y en muchas veces generando la muerte de los mismos; datos que se podrían complementar con lo descrito por González y Aportella (2001) en diferentes especies de crustáceos.

Tabla 1. Porcentaje promedio de letalidad del colorante alimentario Rojo 40 de los diferentes tratamientos en nauplios de Artemia salina.

\begin{tabular}{|c|c|c|c|c|}
\hline \multirow{2}{*}{ TRATAMIENTOS } & \multicolumn{3}{|c|}{ LETALIDAD (\%) } & \multirow{2}{*}{$x$} \\
\hline & R1 & R2 & R3 & \\
\hline TO $(0,0 \mathrm{mg} / \mathrm{L})$ & 0 & 0 & 0 & 0 \\
\hline T1 (3,125 mg/L) & 65 & 55 & 60 & 60 \\
\hline T2 (15.625 mg/L) & 100 & 100 & 100 & 100 \\
\hline T3 (31.25 mg/L) & 100 & 100 & 100 & 100 \\
\hline
\end{tabular}

Figura.1 Mortalidad de nauplios de Artemia salina según concentración de colorante Rojo 40

Cabe destacar al mismo tiempo, que estas concentraciones son empleadas tomando como valor la ingesta diaria IDA del Rojo $40(0-7,0 \mathrm{mg} / \mathrm{kg}$ de peso corporal-día) según FDA (JECFA, 2011) así como la dosis máxima que deben poseer diversos alimentos procesados $(300 \mathrm{mg} / \mathrm{Kg})$ y bebidas (entre $20 \mathrm{mg} / \mathrm{L}-30 \mathrm{mg} / \mathrm{L})$ según Codex Alimentarius (Shanmugasundaram et al., 2019).

La propuesta de la presente investigación radica en la existencia de reportes que se contradicen con respecto a los efectos en la salud, hay estudios de toxicología genética que mostraron que el Rojo 40 no tiene potencial mutagénico, posiblemente debido al tipo de dosis empleada en el estudio (Santillán et al., 2018; Brown et al., 1978; Andrioli et al., 2006); mientras que Tsuda et al. (2001) afirmaron el hecho de que el Rojo 40 puede causar daño a nivel de ADN in vivo; basados en su estudio con Electroforesis en Gel de Célula Única (SCGE), dando positi- 
vo a la presencia de mutaciones a nivel celular en muestras de estómago glandular, pulmones y colon de ratones tratados con este colorante.

Tabla 2. Análisis de Varianza (ANOVA) en la concentración experimental del rojo 40.

\begin{tabular}{lccccc}
\multicolumn{1}{c}{ F.V. } & SC & $\mathbf{g l}$ & $\mathbf{C M}$ & $\mathbf{F}$ & p-valor \\
\hline LETALIDAD & 20156.25 & 3 & 6718.75 & 3225.00 & $<0.0001$ \\
TRATAMIENTOS & 20156.25 & 3 & 6718.75 & 3225.00 & $<0.0002$ \\
ERROR & 16.67 & 8 & 2.08 & & \\
\hline TOTAL & 20172.92 & 11 & & &
\end{tabular}

Tabla 3. Test de Tukey para determinación de cifras significativas.

\begin{tabular}{ccccccc} 
TRATAMIENTOS & MEDIAS & N & E.E. & & & \\
\hline 0.000 & 0.00 & 3 & 0.83 & A & & \\
3.125 & 58.33 & 3 & 0.83 & & B & \\
15.625 & 100.00 & 3 & 0.83 & & & C \\
31.250 & 100.00 & 3 & 0.83 & & & C \\
\hline
\end{tabular}

Medias con letra común no son significativamente diferentes $(\mathrm{p}>0.05)$

Las larvas de Artemia sp. se consideran como biosensores y reportan la diferente sensibilidad frente a los biocidas ensayados, la que varía dependiendo de la especie con la que se compare (Shazili y Pascoe, 1986; Williams et al., 1986); aunque esta también puede variar dependiendo de los distintos estadios de desarrollo; de allí que investigadores como Hutchinson et al. (1998) y Bateman et al. (2004), afirman que, los estudios de toxicidad normalizados son usualmente realizados con organismos neonatos o juveniles, ya que se les reconoce más sensibilidad, asegurando así la obtención de resultados que establezcan niveles de seguridad mayores frente a las exposiciones; estableciendo así la siguiente relación de uso de estos especímenes en pruebas de toxicidad: formas larvarias $>$ formas juveniles $>$ formas adultas.

Lo descrito en el párrafo anterior se afianza, en el hecho de que existen investigadores como Bustos-Obregón y Vargas (2010), que ratifican el empleo de estos especímenes en estudios de selección de sitios en cultivos marinos (valorando la calidad del sedimento) y en la evaluación de la toxicidad en ecosistemas marinos.

\section{CONCLUSIONES}

El colorante alimentario rojo 40 presentó efecto citotóxico en nauplios de Artemia salina Leach. Se presentó una letalidad del $100 \%$ a las concentraciones de $15.625 \mathrm{mg} / \mathrm{L}$ y 31.25 $\mathrm{mg} / \mathrm{L}$; con una DL 50 de $2.6 \mathrm{mg} / \mathrm{ml}$.

\section{REVISIÓN BIBLIOGRÁFICAS}

Abdellah, A., Abdelmonem, M., Emam, A. y Sherif, H. (2015). Physiological Effects Of Some Artificial And Natural Food Coloring On Young Male Albino Rats. Journal of Food Technology Research, 2(2): 21-32.

Amaringo, F. y del Socorro, A. (2018). Adsorción de rojo 40 sobre cascarilla de arroz: determinación del equilibrio, cinética y termodinámica. Tecnura 22(56): 13-28

Andrioli, N., Wulff, A. y Mudry, M. (2006). Allium cepa como monitor de toxicidad y genotoxicidad de metronidazol. Theoria 15(2): 9-16.

ASPEC. (2009). Peligrosa tartrazina en Inka Kola, productos Costa y otros. Con nuestro Perú. Recuperado de http://www.connuestroperu.com/consumidor/20/8367-peligrosa-tartrazina-eninka-kola-productos-costa-y-otros.

Badui-Dergal,S.(2006). Químicadelosalimentos. Pearson Educación, México, 4 (9) 403pp.

Bateman, B., Warner, J., Hutchinson, E., Dean, T., Rowlandson, P., Gant, C., Grundy, J., Fitzgerald, C. y Stevenson, J. (2004). The effects of a double blind, placebo controlled, artificial food colourings and benzoa- 
te preservative challenge on hyperactivity in a general population sample of preschool children. Arch Dis Child 89 (6): 506-511.

Bustos, O. y Vargas, A. (2010). Chronic toxicity bioassay with populations of the crustacean Artemia salina exposed to the organophosphate diazinon. Revista NCBI 43(3): 357-362.

Brown, J., Roehm, G. y Brown, R. (1978). Mutagenicity testing of certified food colors and related azo, xanthene and triphenylmethane dyes with the salmonella/microsome system. Mutat Res 56: 249-271.

CSPI. (2011). FDA Urged Prohibid Carcinogenic "Caramel coloring". Center for Science in the Public interest. Recuperado de:https://cspinet.org/new/201102161.html.

DIGESA. (2013). Evalúan 300 productos que contienen tartrazina. Con nuestro Perú. Recuperado de http://www.connuestroperu. com/consumidor/20/34301-evaluan-300-productos-que-contienen-tartrazina

González, Y. y Aportela, P. (2001). Determinación de la Toxicidad aguda del dicromato de potasio en larvas de Artemia salina. Anuario Toxicología 1(1): 4-108.

Hutchinson, T., Solbe, J. y Kloepper-Sams, P. (1998). Analysis of the ECETOC aquatic toxicity (EAT) database.III-Comparative toxicity of chemical substances to different life stages of aquatic organisms. Chemosphere 36:129-142.

Martine, P., Gérard, J., Mostafa, O. E. y Jean, M. (2009). Lack of genotoxic effect of food dyes amaranth, sunset yellow and tartrazine and their metabolites in the gut micronucleus assay in mice. Food and Chemical Toxicology 47(2): 443-448. McCann, D., Barrett, A., Cooper, A., Crumpler, D., Dalen, L., Grimshaw, K., Kitchin, E., Lok, K., Porteous, L., Prince, E., Sonuga-Barke, E.,

Warner, J. y Stevenson, J. (2007). Food additives and hyperactive behavior in 3-year-old and 8/9-year-old children in the community: a randomized, double-blinded, placebo-controlled trial. Lancet 370 (9598):1560- 1567.

Okefor, S., Obonga, W., Ezeokonkwo, M., Nurudeen, J., Orovwigho, U. y Ahiabuike, J. (2016). Journal of Pharmaceutical and Biosciences. 4(4), 01-11.

Pino, O. y Jorge, F. (2010). ENSAYO DE Artemia: Útil Herramienta De Trabajo Para Ecotoxicólogos Y Químicos De Productos Naturales. Rev. Protección Veg. 22 (1): 34-43.

Shanmugasundaram, P., Bavenro, B. y Rujaswini T. (2019). A Review on Food Coloring Agents - Safe or Unsafe?. Research J. Pharm. and Tech. 12(5):2503-2505.

Santillán, F. y Maza, I. (2018). Remoción de colorantes azoicos rojo allura (rojo 40) mediante el uso de perlas de quitosano magnetizadas en medio acuoso. Revista de la Sociedad Química del Perú 84(1): 18-26.

Secco, A. (1994). Colorantes Sintéti$\cos$ y Naturales para Uso en Alimentos. Revista de la Sociedad Chilena de Tecnología de Alimentos 19(3): 31-39

Shazili, N. y Pascoe, D.( 1986). Variable sensitivity of rainbow trout (Salmo gairdneri) eggs and alevins to heavy metals. Bulletin Environ- 
mental Contamination Toxicology 36:468-474.

Tripathy, S.; Rao, D. (2015). Mitotic aberrations induced by orange red (a food additive dye) as a potential genotoxicant on root tip cells of onion (Allium cepa L.) International Food Research Journal 22(1): 383-392.

Tsuda, S., Murakami, M., Matsusaka, M., Kano, T. y Sasaki, T. (2001). DNA damage induced by Red Food Dyes Orally Administered to pregnant and Male Mice. Toxicological Sciences 61:91-99. 